CONCLUSIONS: A clinician-based global assessment indicated ongoing, meaningful TD improvements in adults who received once-daily VBZ in the current study. In participants treated for $>1$ year, continued patient satisfaction rates with VBZ were high.

Funding Acknowledgements: Neurocrine Biosciences, Inc.

39

\section{Long-term Safety and Tolerability of Once-Daily Valbenazine in Patients with Tardive Dyskinesia}

Stephen R. Marder, MD'; Martha Sajatovic, MD'; Dan Michel, PharmD 3 ; Joshua Burke, MS ${ }^{4}$; Khody Farahmand, PharmD ${ }^{5}$; and Scott Siegert, PharmD ${ }^{6}$

${ }^{1}$ Professor, Psychiatry and Biobehavioral Sciences, University of California Los Angeles, David Geffen School of Medicine, Los Angeles, CA

${ }^{2}$ Director, Neurological and Behavioral Outcomes Center, University Hospitals Cleveland Medical Center and Professor, Psychiatry, Case Western Reserve University School of Medicine, Cleveland, $\mathrm{OH}$

${ }^{3}$ Senior Medical Liaison, Neurocrine Biosciences, Inc., San Diego, CA

${ }^{4}$ Director, Biostatistics and Data Management, Neurocrine Biosciences, Inc., San Diego, CA

${ }^{5}$ Director, Medical Communications, Neurocrine Biosciences, Inc., San Diego, CA

${ }^{6}$ Executive Director, Medical Affairs, Neurocrine Biosciences, Inc., San Diego, CA

ABSTRACT: Objective: To evaluate the long-term safety and tolerability of once-dailyvalbenazine in adults with tardive dyskinesia (TD).

METHODS: Data were pooled from KINECT 3 (NCT02274558: 6-week double-blind placebocontrolled period, followed by a 42 -week double-blind extension and 4-week drug-free washout) and KINECT 4 (NCT02405091: 48-week open-label treatment period and 4-week drug-free washout). KINECT $3 / 4$ study completers could enroll in a subsequent rollover study (NCT02736955: up to 72 weeks of open-label treatment or until valbenazine became commercial available); data from this study were described separately for this analysis. Valbenazine dose groups (40 and $80 \mathrm{mg}$ ) were pooled for analysis. Safety assessments included treatment-emergent adverse events (TEAEs) and the Columbia-Suicide Severity Rating Scale (C-SSRS). Psychiatric status was assessed in KINECT 3 and KINECT 4 using the following measures: Positive and Negative Syndrome Scale (PANSS) total score and Calgary Depression Scale for Schizophrenia (CDSS) in participants with schizophrenia/schizoaffective disorder; Montgomery-Åsberg Depression Rating Scale (MADRS) and
Young Mania Rating Scale (YMRS) in participants with a mood disorder.

RESULTS: Analyses included 304 KINECT 3/4 participants and 160 rollover participants. In KINECT 3/4, the summary of TEAEs was as follows: any TEAE (71.7\%), serious TEAE (16.8\%), and discontinuation due to TEAE (15.5\%). TEAEs reported in $\geq 5 \%$ of all KINECT $3 / 4$ participants were headache $(8.9 \%)$, urinary tract infection $(8.9 \%)$, somnolence $(7.9 \%)$, fatigue $(6.3 \%)$, dizziness $(5.9 \%)$, and suicidal ideation $(5.6 \%)$. The summary of TEAEs from the rollover study was as follows: any TEAE (53.1\%), serious TEAE (10.0\%), and discontinuation due to TEAE (5.6\%). The most common TEAEs in the rollover study were back pain and urinary tract infection (4.4\%, each); no TEAE was reported in $\geq 5 \%$ of participants. Minimal changes in psychiatric status were observed in KINECT $3 / 4$, as indicated by mean score changes from baseline to Week 48 in participants with schizophrenia/schizoaffective disorder (PANSS total, -3.2; CDSS total, -0.5) or a mood disorder (MADRS total, 0.3; YMRS total, -1.0). Over one-third of study participants had a lifetime history of suicidal ideation or behavior (KINECT 3/4, 41\%; rollover, 38\%). Most participants had no C-SSRS suicidal ideation at study baseline; of these, $>90 \%$ had no emergence of suicidal ideation at any time during the study (KINECT $3 / 4,93 \%$ [276/296]; rollover, 98\% [153/156]).

CONCLUSIONS: Valbenazine was well tolerated and no unexpected safety signals were found in adults who received $>1$ year of once-daily treatment. Psychiatric stability was maintained, and few participants experienced any emergence of suicidal ideation during the studies despite $35-40 \%$ having a lifetime history of suicidality. These results indicate that once-daily valbenazine may be an appropriate treatment for the longterm management of TD.

Funding Acknowledgements: Neurocrine Biosciences, Inc.

\section{0}

\section{Pseudo Cranial Nerve I Dysfunction: Subjective Hyposmia and Subjective Hypogeusia but Normosmia and Normogeusia - 3 cases}

\section{Kristal Benskin' ${ }^{\text {; }}$ and Alan R. Hirsch, M.D.,F.A.C.P ${ }^{2}$}

${ }^{1}$ Medical Student, American University of Barbados School of Medicine, Bridgetown, Barbados, West Indies

${ }^{2}$ Smell and Taste Treatment and Research Foundation Ltd., Chicago, Illinois

ABSTRACT: INTRODUCTION: Hyposmia refers to reduced ability to smell and hypogeusia is a partial loss 
of the ability to taste (Hummel, Basile, \& Huttenbrink, 2016). Complaints of hyposmia and hypogeusia in the presence of normosmia and normogeusia has not heretofore been described. Three such cases are presented.

OBJECTIVE: To explore the complaints of reduced smell and taste with normal objective olfaction and gustation.

METHOD: All patients were given screening tests for smell and taste and obtained scores consistent with normosmia and normogeusia. The 12-item version of the Brief Smell Identification Test (B-SIT), using the odorants banana, chocolate, cinnamon, gasoline, lemon, onion, paint thinner, pineapple, rose, soap, smoke and turpentine was used. The Retronasal Olfactory Test was used to determine their perception of flavour and the Proplythiouracil Disc Taste Test used for gustation. Each patient also underwent a complete physical and neurological examination with any abnormalities mentioned.

RESULTS: Case 1: This 53 year old female, 8 months prior to presentation, developed the flu followed by the inability to taste any foods and differentiate between smells, with everything smelling bitter.

Results: Chemosensory testing: Olfaction: Brief Smell Identification Test (B-SIT): 10 (normosmia). Retronasal Olfactory Test: Retronasal Smell Index: 9 (normal). Gustation: Propylthiouracil Disc Taste Test: 7 (normal). Case 2: This 86 year old female, 6 months prior to presentation, developed reduced taste, of gradual onset, to the point upon presentation was only $10 \%$ normal. She was able to taste lemons but very little else.

Results: B-SIT: 9 (normosmia). Retronasal Smell Index: 10 (normal). Propylthiouracil Disc Taste Test: 10 (normogeusia).

Case 3: This 63 year old female was nasute until 3 months prior to presentation, when she developed an upper respiratory infection, followed by loss of smell and taste to $20 \%$ of normal.

Results: B-SIT: 10 (normosmia). Retronasal Smell Index: 7 (normal). Propylthiouracil Disc Taste Test: 10 (normogeusia).

CONCLUSION: Discordance between subjective and objective findings may be due to the wide distribution of normal in the general population in olfactory ability. The associated reduction in retronasal smell may then be interpreted by the patient as reduced ability to taste. Alternatively, complaints of hyposmia and hypogeusiamay be due to a non-organic need such as malingering or psychosomatic illness, or could represent a primary defect in the cortical integration of smell and taste, interpreted as reduction in perceived flavor, yet the primary sensory neurons and threshold as tested would appear to be normal. This disparity possibly indicates that the testing modalities are too insensitive to demonstrate more subtle sensory perception findings, and suggests the need for more refined testing methods for smell and taste.

\section{1}

\section{A Modified Delphi Consensus Approach to Clinical Guidelines for Tardive Dyskinesia}

Stanley N. Caroff, $\mathrm{MD}^{\prime}$; Leslie Citrome, $\mathrm{MD}, \mathrm{MPH}^{2}$; Jonathan Meyer, $\mathrm{MD}^{3}$; Kimberly Riggs, $\mathrm{MPH}^{4}$; Martha Sajatovic, $M D^{5}$; Leslie Lundt, $M D^{6}$; and Terence A. Ketter, $M D^{7}$

${ }^{1}$ Emeritus Professor CE of Psychiatry, Corporal Michael J. Crescenz Veterans Affairs Medical Center and the Perelman School of Medicine at University of Pennsylvania, Philadelphia, PA

${ }^{2}$ Clinical Professor of Psychiatry and Behavioral Sciences, New York Medical College, Valhalla, NY

${ }^{3}$ Clinical Professor of Psychiatry, UC San Diego School of Medicine, San Diego, CA

${ }^{4}$ Associate Director, Xcenda, Palm Harbor, FL

${ }^{5}$ Professor, Psychiatry, Case Western Reserve University School of Medicine, Cleveland, $\mathrm{OH}$

${ }^{6}$ Medical Director, Neurocrine Biosciences, Inc.

${ }^{7}$ Professor of Psychiatry and Behavioral Sciences, Stanford University School of Medicine, Stanford, CA

ABSTRACT: Objective: Vesicular monoamine transporter 2 (VMAT2) inhibitors are the first class of drugs approved to treat tardive dyskinesia (TD). With the recent approval of these medications, a modified Delphi process was implemented to address the need for updated clinical guidelines for TD screening, diagnosis, and treatment.

METHODS: A Steering Committee of 11 TD experts met in a Nominal Group meeting format to discuss/prioritize questions to be addressed about TD and identify individuals to be invited to serve as Delphi survey panelists. Two survey rounds were conducted anonymously; responses were collected, collated, and analyzed. Respondent agreement was defined a priori by the Steering Committee as unanimous $(100 \%)$, consensus (75-99\%), or majority (50-74\%). For questions using a 5 -point Likert scale, agreement was based on percentage of respondents choosing $\geq 4$ ("agree completely" or "agree"). Round 1 survey included questions on TD screening, diagnosis, and treatment. Round 2 questions were refined per panelist feedback and excluded Round 1 questions with $<25 \%$ agreement (deemed unlikely to achieve consensus) and some questions that already achieved consensus (>75\% agreement).

RESULTS: Online surveys were sent to 60 individuals; 29 agreed to participate as panelists (23 psychiatrists; 6 neurologists). Respondents unanimously agreed $(100 \%)$ 